\title{
Survey Evaluation of an Approach for Dynamic Software Product Lines
}

\author{
Gabriela Guedes $^{1}$, Carla Silva ${ }^{2}$, Monique Soares ${ }^{3}$ \\ ${ }^{1}$ Instituto Federal da Paraíba (IFPB) - Campus Guarabira \\ Rodovia PB 057 - KM-02 - 58.200-000 - Guarabira - PB - Brazil \\ ${ }^{2}$ Centro de Informática- Universidade Federal de Pernambuco (CIn-UFPE) \\ Recife - PE - Brazil \\ ${ }^{3}$ Escribo - Recife - PE - Brazil \\ \{gabriela.souza@ifpb.edu.br, ctlls@cin.ufpe.br, \\ monique.avalon@gmail.com
}

\begin{abstract}
In Dynamic Software Product Lines (DSPLs) product configuration occurs at runtime. Modelling variability and configuring the product in DSPLs should consider the context and non-functional requirements (NFRs) satisfaction. ConG4DaS (Contextual Goal models For Dynamic Software product lines) is a Requirements Engineering (RE) approach for DSPL which supports (i) modelling of variability with goals, NFRs, contexts and the relationship be-tween them; and (ii) offers a product configuration based on contexts, NFRs and their priority and interactions. In this paper, we present a survey evaluation of ConG4DaS with RE and DSPL researchers in regard to the perceived usefulness. In the survey, both RE and DSPL researchers perceived ConG4DaS as useful for modelling and configuring DSPLs. However, the RE group gave more positive answers than the DSPL group.
\end{abstract}

\section{Introduction}

Software Product Lines (SPLs) offer an effective way to develop a series of similar and domain-specific systems that share a common core, but may have some variable characteristics [Clements and Northrop 2001]. SPL approaches are capable of modelling variability in terms of variation points (what may vary in the software) and variants (possible variations for the variation point), and, when a new product is required, they provide means for configuring variability (bind variation points to a variant).

Dynamic Software Product Lines (DSPLs) [Hallsteinsen et al. 2008] extend these SPL's capabilities to runtime, using SPL techniques for developing adaptive systems. According to Capilla et al. (2014), a DSPL should possess the following properties:

Cadernos do IME - Série Informática

e-ISSN: 2317-2193 (online)

DOI: 10.12957/cadinf.2020.56857 
- Runtime variability support and management: a DSPL should allow the analysis and reconfiguration of the system's variability at runtime;

- Multiple and dynamic binding: when the system adapts its properties to a new context, features can be bound several times and at different binding times;

- Context-awareness and self-adaptation for autonomic behaviour: DSPLs should handle context in-formation that is used to dynamically select new system options depending on the environment conditions.

Thus, a Requirements Engineering (RE) approach for DSPLs should provide models for capturing variability and a way for configuring this variability taking into account the conditions that trigger and drive the adaptation. Those conditions may be related to the external environment where the DSPL operates, i.e., the context, or to the non-functional requirements (NFRs) it should satisfy, which may be operationalized as internal system properties or quality constraints or the quality of service (QoS) it provides.

We proposed ConG4DaS (Contextual Goal models for Dynamic Software product lines) [Guedes, Silva and Soares 2017], a Requirements Engineering (RE) approach for DSPLs that captures the DSPL's variability by using goals, NFRs (modelled as softgoals), contexts and the relationships among them. In the configuration process, the available configurations for the given context are ranked according to the priority of the softgoals they satisfy, and the best-ranked configuration is selected.

The objective of this paper is to present the results of a survey with RE and DSPL researchers with respect to ConG4DaS' perceived usefulness. In this survey, RE and DSPL researchers were invited to read ConG4DaS description presented in [Guedes 2020] and answer an online questionnaire about the approach's perceived usefulness.

The remainder of this paper is organized as follows. Section 2 presents the background of this work. In section 3 we present an overview of ConG4DaS. Section 4 contains the results of the survey. Then, we conclude this paper in Section 5.

\section{Background}

This section presents the main concepts of the areas related to this work, which includes Dynamic Software Product Lines and Goal-Oriented Requirements Engineering.

\subsection{Dynamic Software Product Lines}

A Software Product Line may be seen as a family of software systems that share many characteristics, but may vary in others. According to Pohl, Böckle and van der Linden (2005), Software Product Line Engineering (SPLE) is a paradigm to develop software systems using platforms and mass customization. They argue that the use of SPLE brings improvements in cost, quality, productivity and time to market. The same authors proposed an SPLE framework that consists of two separate processes:

- Domain Engineering (DE): responsible for establishing the reusable platform and defining the commonality and the variability of the product line. During DE, variability is defined in variability models [Pohl, Böckle and van der Linden 2005]; 
- Application Engineering (AE): responsible for deriving product line applications from the platform established in domain engineering. In $\mathrm{AE}$, for deriving a new product, the variability must be configured.

When a product of an SPL is delivered, all of its variation points (VPs) have been bound at design time, the product can no longer change to a new configuration. However, when VPs may be bound at runtime and the product is capable of changing its configuration, we call it a Dynamic Software Product Line (DSPL). In DSPLs, variability configuration occurs at runtime, i.e., the $\mathrm{AE}$ process is executed at runtime if a new configuration is needed.

For Hallsteinsen et al. (2008), DSPL can be seen as one among several approach-es to building self-adapting/managing/healing systems. They provide a way of modelling adaptive systems by using SPL variability management techniques to deal with the variable functionality this kind of systems may present at runtime. Thus, the self-adaptive system (SAS) itself is seen as a DSPL and every possible configuration of the SAS could be seen as a product of the DSPL [Bencomo et al. 2008].

Therefore, when we refer to DSPLs, "we are not necessarily dealing with an entire product line in the traditional sense, but we might perceive the DSPL to be a single system, adapting its behaviour when variability is rebound during operation" [Hinchey, Park and Schmid 2012].

Thus, the DSPL has to monitor the environment and/or its own properties to detect when it should adapt itself. So, its variability should be associated to the environment context or system properties in order to select a variant to cope with current situation.

\subsection{Goal-Oriented Requirements Engineering}

Requirements define what the system should do, describing the services that it provides and the constraints on its operation [Sommerville 2010]. Requirements Engineering (RE) is the software development phase that involves discovering, documenting, and maintaining a set of requirements of a software system [Kotonya and Sommerville 1998].

In Goal-Oriented Requirements Engineering (GORE) approaches, requirements are specified in terms of goals. A goal is an objective the system should achieve and it may cover a functional or non-functional concern [Lamsweerde 2001]. Some of the advantages of using goals are [Lamsweerde 2001] [Liaskos et al. 2006]:

- Goals provide precise criterion for requirements pertinence. A requirement is pertinent if it contributes to the satisfaction of at least one stakeholders' goal;

- Goals may be formulated at different levels of abstraction. Goal refinement provides a mechanism for structuring complex requirements;

- Alternative goal refinements allow the identification of requirements variability, by capturing alternative ways for achieving stakeholders' goals.

In DSPLs, goal-based approaches adopt more abstract representations of the decision model, allowing for analysis and planning at runtime [Bencomo, Hallsteinsen and Almeida 2012]. One particular GORE approach that has been used as basis for many works is the $i^{*}$ (iStar) framework [Yu et al. 2011]. In this framework, participants 
of the organizational environment of the system-to-be and the system itself are modelled as organizational actors that depend on one another to achieve their goals. Our work has a variability model based on an extension of iStar.

\section{ConG4DaS}

ConG4DaS (Contextual Goal models for Dynamic Software product lines) [Guedes, Silva and Soares 2017] is a Requirement Engineering process for developing Dynamic Software Product Lines. It has two processes: the Domain Engineering and the Application Engineering.

When defining ConG4DaS, our goal was to provide an approach that allows the DSPL to adapt itself to context, but maximizing the NFRs' (which are modelled as softgoals) satisfaction as much as possible in the given context. To accomplish this, ConG4DaS requirements models provide a way to capture how the context affects softgoals priorities and a manner to model how an element's contribution to a softgoal may change with the context. Then, this information is used during the configuration process, so the variants are selected based on how they contribute to the softgoals, giving preference to those with higher priority. In the next subsections, we briefly describe each sub-process of ConG4DaS. A complete description of all activities can be found in ConG4DaS website [Guedes 2020].

\subsection{Running Example: Smart Home}

In order to illustrate the application of ConG4DaS, in the next sections we use the Smart Home example, which was adapted from the example presented by Pimentel et al. (2012) and Ali, Dalpiaz and Giorgini (2013).

In Smart Home, the following variations are possible: (1) to refresh air inside it, the Smart Home may open the windows, if it is not raining, or turn on the ventilator; (2) the Smart Home should also provide meal suggestions for its tenant. It may suggest a home cooked meal, if there is enough food in stock, or suggest a restaurant order; (3) the Smart Home controls the lights inside the house and it may turn on the lights only in occupied rooms or, when there is no one home, simulate occupancy by turning the lights on different rooms; (4) the Smart Home also controls the food stock, warning the tenant when it is low.

\subsection{Domain Engineering}

The DE process of ConG4DaS uses $i$-orthogonal [Lima 2011], an iStar extension that includes cardinality and context annotations, as the variability model. The first three activities in the process are mandatory and concern the creation of the $i^{*}$-orthogonal and context models, which are used in the AE process. The DE process also includes an optional sub-process for deriving the DSPL's feature model and use case scenarios stubs. Figure 1 depicts the activities of ConG4DaS DE process.

For most activities, we have proposed steps that must be followed in order to create the requirements models. The steps provided in the "Specify Context Model" and "Represent Variability" activities are not automated, since they only provide guidance on how to represent variability in the $i^{*}$-orthogonal and context models. The creation of this models depends on the requirements elicitation, which is not covered by our approach. However, the steps of the "Create Option-al Artefacts" sub-process are 
automated by the GCL-Tool (Goal and Context for product Line - Tool) [Varela 2015], which also provides editors for the $i^{*}$-orthogonal and context models. Unfortunately, due to the lack of space, we do not describe the steps of each activity in this paper, but they can be found in ConG4DaS website [Guedes 2020].

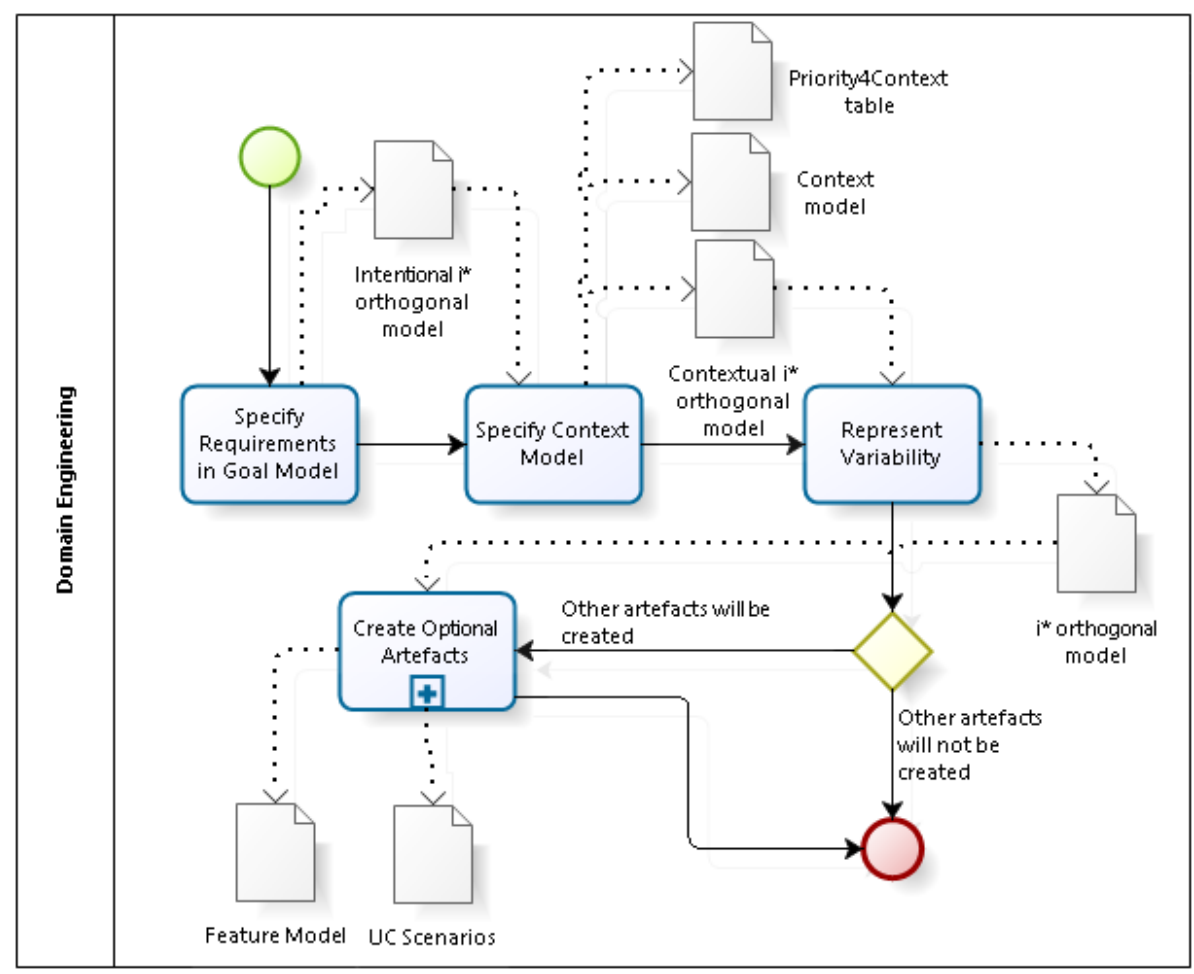

Figure 1. DE process of ConG4DaS

Specify Requirements in Goal Model. The purpose of this activity is to create the initial $\mathrm{i}^{*}$-orthogonal model, that does not contain any context annotation or cardinality in the variation points (VPs). For creating the iStar model, the domain analyst may use any requirements elicitation technique or one of the specific approaches for elaborating iStar models, such as PRiM (Process Reengineering i* Method) [Grau, Franch and Maiden 2008].

Specify Context Model. This activity concerns the construction of the context model and relating it to the $i^{*}$-orthogonal model. There are three steps in this activity to guide the creation of the context model, the annotation of the goal model and the creation of the Priority4Context table. The context model uses the notation proposed in [Ali, Dalpiaz and Giorgini 2013], which decomposes contexts in statements or facts until all leaf nodes are facts. Each fact specifies the conditions in which it is true. When the goal model is annotated with contexts, it means that the relationships and elements with context annotations are only available in the given context. Finally, the Priority4Context table is used to define the priority of softgoals in each context.

The ConG4DAS context model of Smart Home is shown in Figure 2. There are five different contexts: C1 - Not Raining, C2 - Low Food Stock, C3 - There are People Home, C4 - Low Budget and C5 - Night Time. C1 is active when sensors do not detect rain. $C 2$ is active when the food stock at home is lower than $15 \%$. $C 3$ is active when there is at least person inside the house. $C 4$ is active when more than $75 \%$ of the 
monthly budget has been used. $C 5$ is active when it is dark outside. The Priority4Context table is shown in Table 1 . The softgoals' priority was not defined for the contexts $C 1, C 2, C 3$ and $C 5$, which means that they use the default priority.

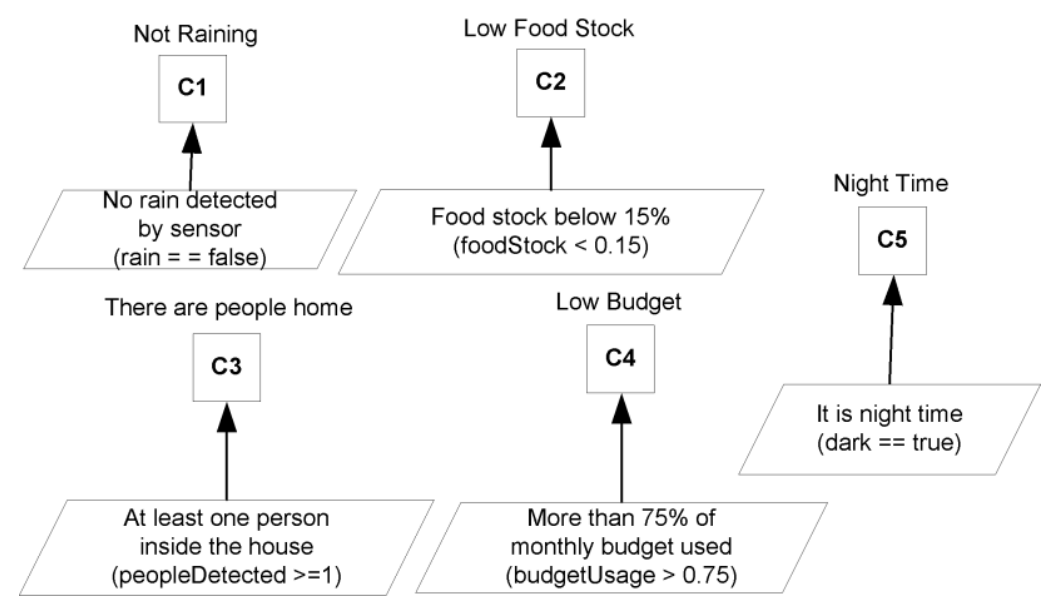

Figure 2. Context model for Smart Home

Table 1. Priority4Context table for Smart Home

\begin{tabular}{l|c|c|c|c|c|c} 
& Default & C1 & C2 & C3 & C4 & C5 \\
\hline Safety (SF) & 10 & - & - & - & 8 & - \\
Save Money (SM) & 8 & - & - & - & 10 & - \\
Energy Efficiency (EE) & 8 & - & - & - & 10 & - \\
$\quad$ Context order & & $5^{\text {th }}$ & $2^{\text {nd }}$ & $4^{\text {th }}$ & $1^{\text {st }}$ & $3^{\text {rd }}$ \\
\hline
\end{tabular}

Represent Variability. In this activity, we identify the variation points (VPs) in the $i^{*}$-orthogonal model by finding the elements that represent features and adding cardinality on them or on their decomposition relationships.

The $\mathrm{i}^{*}$-orthogonal model is depicted in Figure 3. The Tenant actor depends on the Smart Home actor to fulfil the goal dependencies: Refresh Air Inside Home, Control Food Stock, Provide Meal Suggestion, Control Lights and Give Illusion of Being Occupied. Tenant also depends on Smart Home for the softgoal dependencies: Safety, Save Money and Energy Efficiency.

Smart Home's main goal Home Management can be achieved by the Manage Home task. Manage Home is decomposed into the Refresh Air Inside Home, Provide Meal Suggestion and Control Lights goals and into the Control Food Stock task. The Refresh Air Inside Home goal may be achieved by one of the two mutually exclusive tasks Open Windows or Turn on Air Ventilator. Open Windows is only available when $C 1$ is active and it has a help contribution to Energy Efficiency and a hurt contribution to Safety when C5 is active. Turn on Air Ventilator has a hurt contribution to Energy Efficiency and a help contribution to Safety when C5 is active.

Provide Meal Suggestion goal can be achieved by one of the mutually exclusive tasks Suggest Home Cooked Meal, which is only available when $C 2$ is not active, or 
Suggest Restaurant Meal. Suggest Home Cooked Meal has a help contribution to Save Money, and Suggest Restaurant Meal has a hurt contribution to the same softgoal.

Control Lights goal can be achieved by one of the mutually exclusive tasks Occupancy Simulation, which is only available when C3 is not active, or Turn on Lights in Occupied Rooms. Occupancy Simulation has a help contribution to Safety and a hurt contribution to Energy Efficiency, when $C 4$ is active, or a some- contribution to the latter softgoal, when $C 4$ is not active. Turn on Lights in Occupied Rooms has a help contribution to Energy Efficiency and a some- contribution to Safety. Finally, the Control Food Stock task is decomposed into the Update Food Stock and Warn Tenant About Low Food Stock tasks. The latter is executed only when $C 2$ is active.

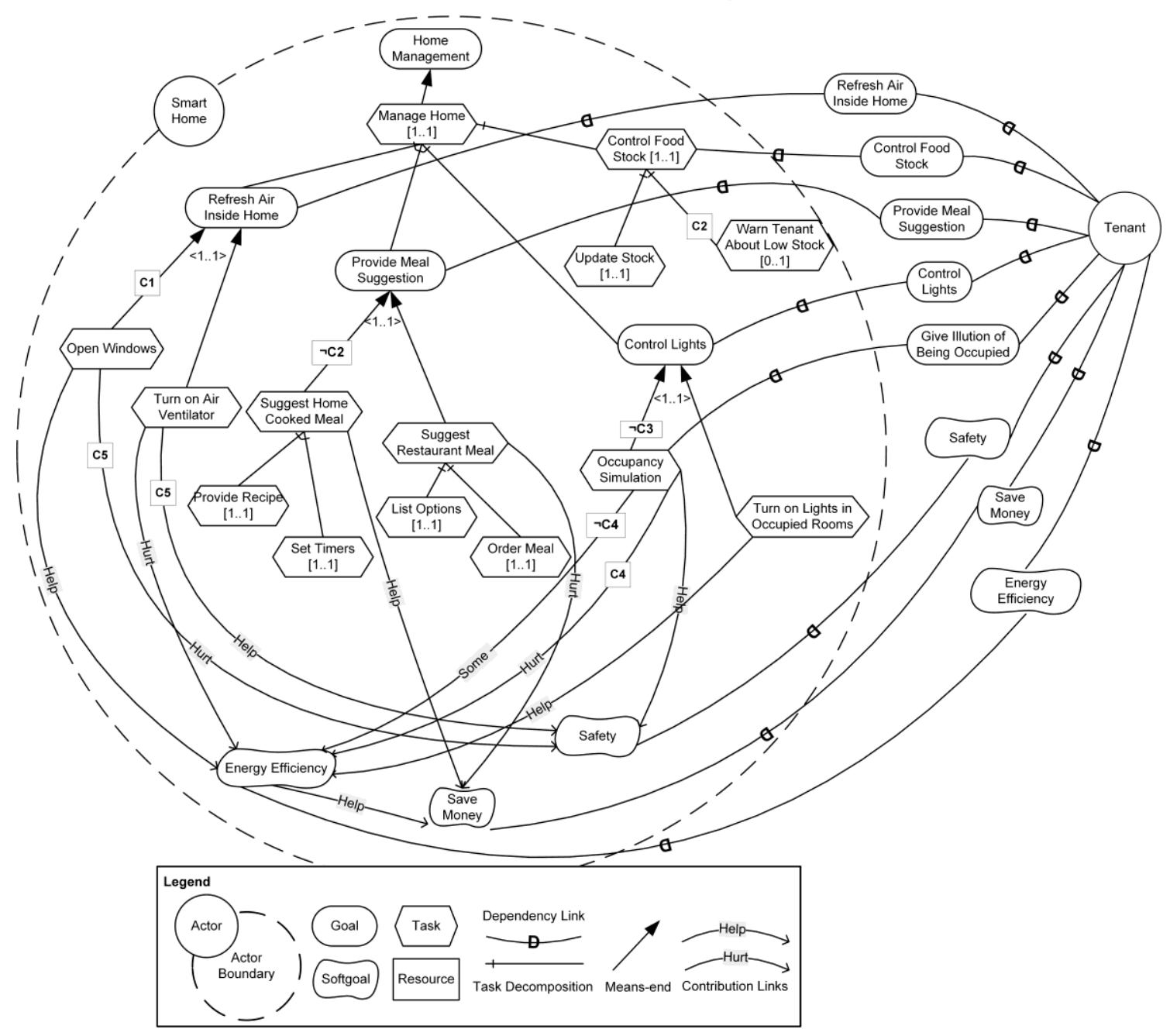

Figure 3. $i^{\star}$-orthogonal model for Smart Home

Create Optional Artefacts. This is an optional sub-process that may be executed by the domain analyst if he wants to improve the requirements description of the DSPL by generating its feature model and use case scenarios. The sub-process is composed by 4 activities: (1) Create Feature Model; (2) Reorganize Feature Model; (3) Create Use Case Scenarios; and (4) Improve Use Case Scenarios. 


\subsection{Application Engineering}

The AE process guides the DSPL's configuration when there is a context change. The first two activities are mandatory and concern the DSPL's configuration based on the $\mathrm{i}^{*}$-orthogonal model. The last activity is optional, because it concerns the configuration of the DSPL's feature model. Figure 4 presents the activities of ConG4DaS AE process.

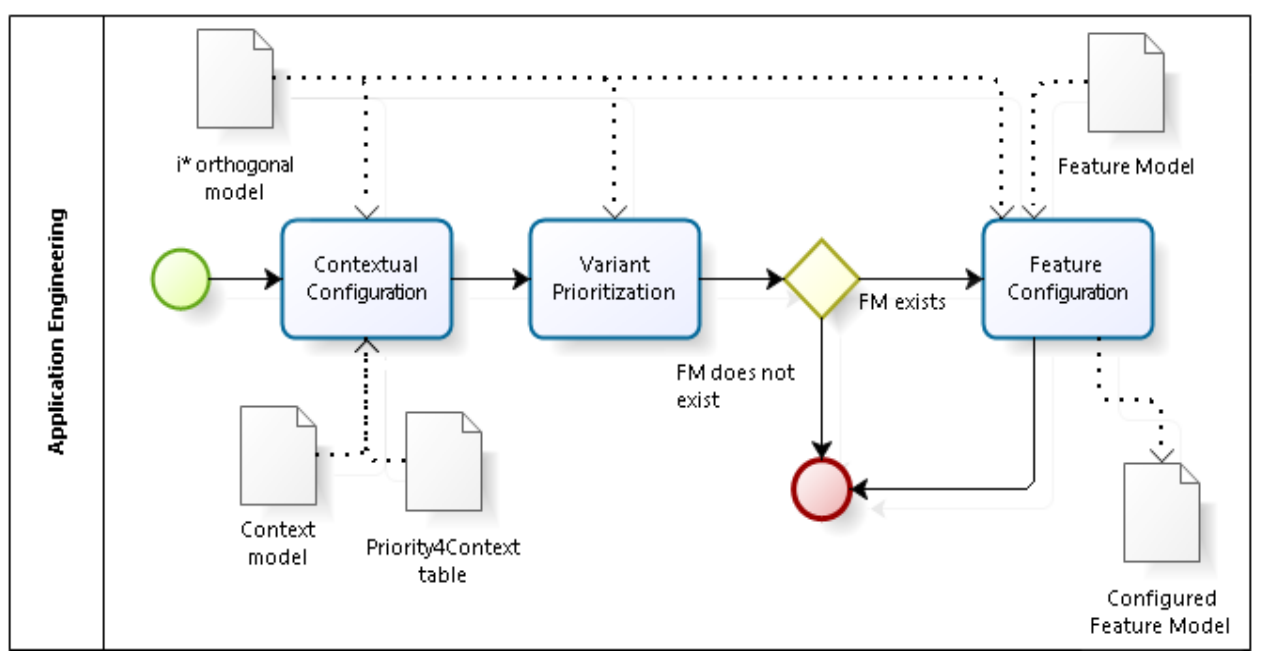

Figure 4. AE process of ConG4DaS

Contextual Configuration. This activity has the objective of discovering possible configurations, in each VP, for current context. In order to do it, the DSPL must monitor the context variables determined by each fact in the context model. For the Smart Home example, let us consider that the context variables have the following values:

$$
\begin{gathered}
\text { rain }=\text { false; foodStock }=0.4 ; \text { peopleDetected }=2 ; \\
\text { budgetUsage }=0.5 ; \text { dark }=\text { false }
\end{gathered}
$$

Analysing the context model (Figure 2), we note that contexts $C 1$ and $C 3$ are active, i.e., it is not raining and there are people at home. Thus, current context is $\mathrm{C} 1 \wedge$ $\neg \mathrm{C} 2 \wedge \mathrm{C} 3 \wedge \neg \mathrm{C} 4 \wedge \neg \mathrm{C} 5$.

When the values of the context variables change in a way that the active contexts also change, then the DSPL must check how the current active contexts interfere on the system's goals. If the values of the context variables from the Smart Home change to:

$$
\begin{gathered}
\text { rain }=\text { true; foodStock }=0.3 ; \text { peopleDetected }=2 ; \\
\text { budgetUsage }=0.5 ; \text { dark }=\text { false }
\end{gathered}
$$

Analysing the context model, we note that $C l$ context is not active anymore because the variable rain now true. However, though the value of foodStock changed from 0.4 to 0.3 , the Food stock below 15\% fact remains false. Therefore, the current context is now $\neg \mathrm{C} 1 \wedge \neg \mathrm{C} 2 \wedge \mathrm{C} 3 \wedge \neg \mathrm{C} 4 \wedge \neg \mathrm{C} 5$. The following steps are defined for configuring the DSPL when the context changes (as in the example above). Since the AE process occurs in execution time, its steps must be implemented, so we present them as pseudo-code. 
AE-Step 1 is for checking whether the new values of the context variables change contexts' activation. Listing 1 contains the pseudo-code for AE-Step 1. For the example above, the change in the context variables, modified Cl's activation, changing context as whole. Therefore, AE-Step 2 would be executed.

In AE-Step 2, annotated relationships in the $i^{*}$-orthogonal model are checked. If the relationship depends on a context that is not active, it is removed from the new configuration. Listing 2 contains the pseudo-code for AE-Step 2.

Analysing the $i^{*}$-orthogonal model (Figure 3), there are some relationships that depend on contexts that are not active in the simulated scenario and, therefore, should be removed: (1) the means-end from Open Windows, which depends on $C 1$; (2) the task decomposition related to Warn Tenant About Low Food Stock, that depends on C2; (3) the means-end from Occupancy Simulation, which depends on $\neg C 3$; (4) the hurt contribution link from Occupancy Simulation to Energy Efficiency, which depends on C4; (5) the hurt contribution link from Open Windows to Safety, that depends on C5; (6) the help contribution link from Turn on Air Ventilator to Safety, that also depends on C5.

\section{Listing 1: AE-Step 1}

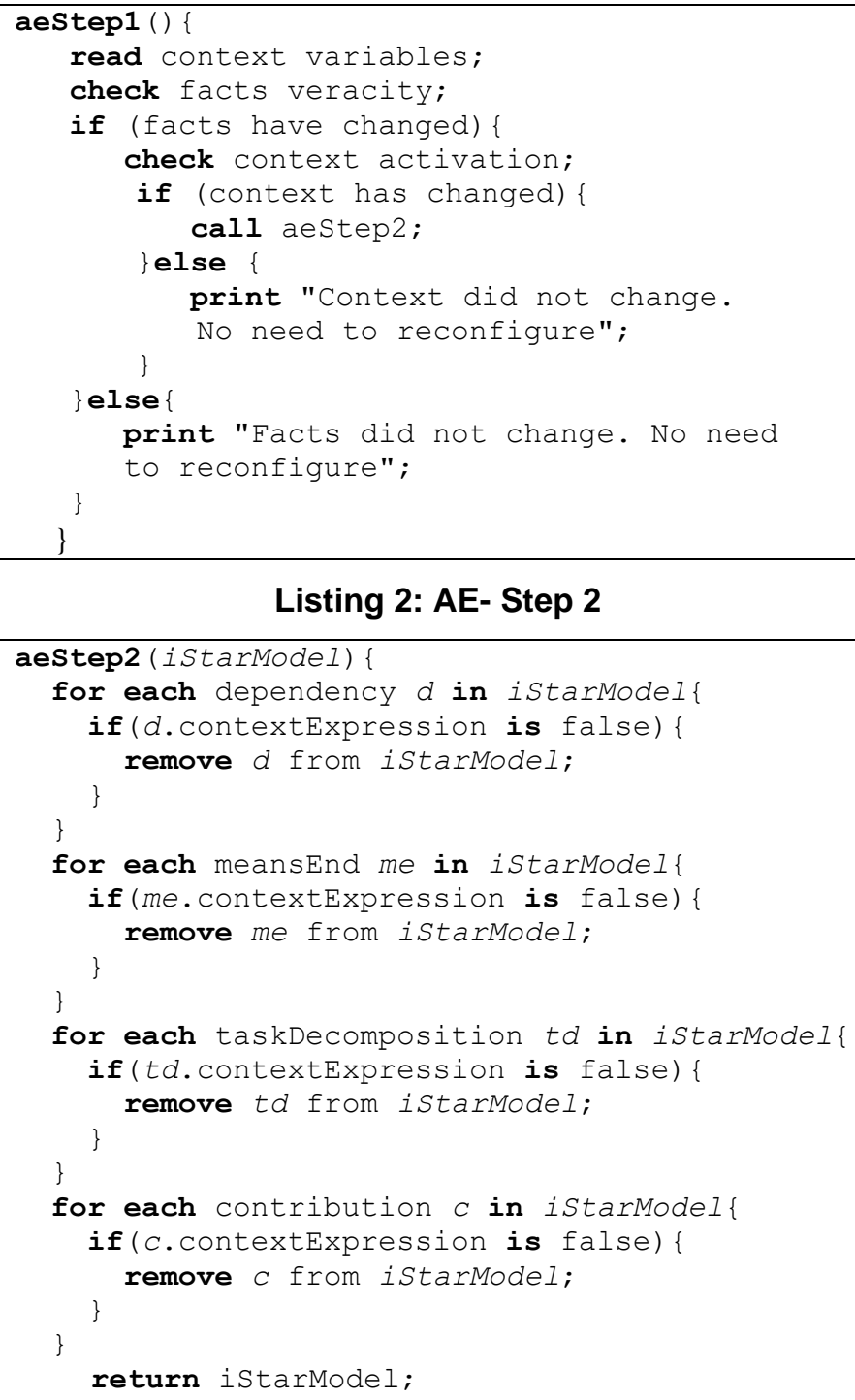

Listing 2: AE- Step 2 
In AE-Step 3, we analyse the variation points in the $\mathrm{i}^{*}$-orthogonal model returned by AE-Step 2. For each VP, we perform the variant prioritization defined in the next activity. Listing 3 contains the pseudo-code for AE- Step 3.

For the simulated context $(\neg \mathrm{C} 1 \wedge \neg \mathrm{C} 2 \wedge \mathrm{C} 3 \wedge \neg \mathrm{C} 4 \wedge \neg \mathrm{C} 5)$, there is only one variation point that has more than one possible variant: Provide Meal Suggestion goal may be achieved by the tasks Suggest Home Cooked Meal, which is available since C2 is not active, or Suggest Restaurant Meal. In order to select one of the variants for the system's reconfiguration, they have to be prioritized, according to the priority function described in the activity Variant Prioritization.

\section{Listing 3: AE- Step 3}

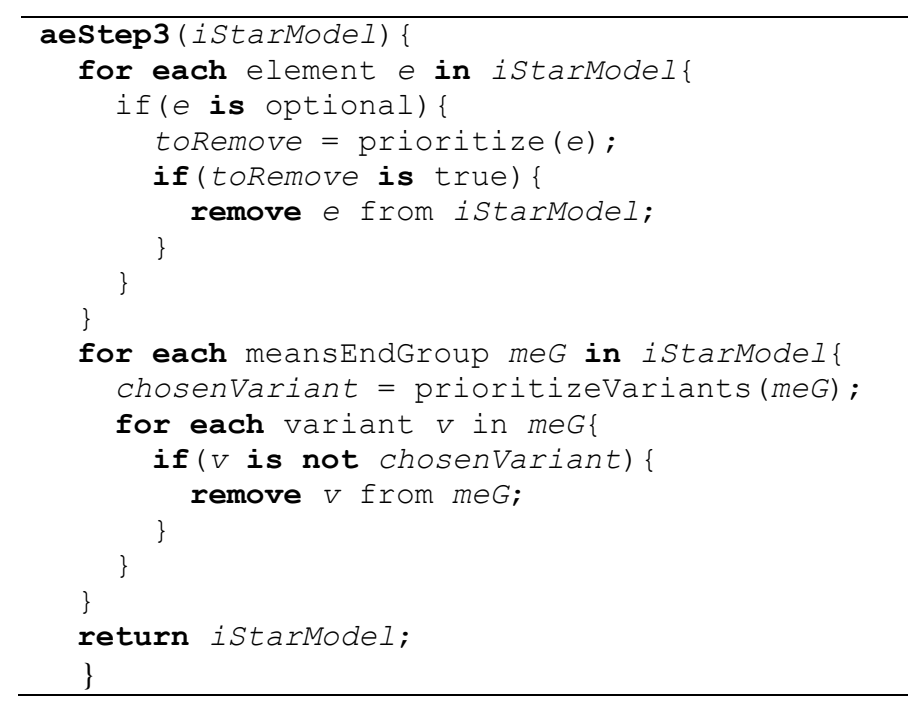

Variant Prioritization. In this activity, those available variants are ranked according to how they contribute to softgoals' satisfaction, taking into account the softgoals' priority for current context. The overall contribution of a variant to the softgoals is calculated according to the following function:

$$
\begin{aligned}
\operatorname{priority}(v) & =\sum_{\mathbf{s g} \in \mathbf{v}} \operatorname{contPos}(v, s g) \times \operatorname{priority}(s g) \\
& -\sum_{\mathbf{s g} \in \mathbf{v}} \operatorname{contNeg}(v, s g) \times \operatorname{priority}(s g)
\end{aligned}
$$

where,

$$
\begin{aligned}
& \operatorname{contPos}(v, s g)=\sum_{c \in t c} \frac{\text { numPosCont }(c, s g)}{\text { totalNumCont }} \times \operatorname{typePosCont}(c) \\
& \operatorname{contNeg}(v, s g)=\sum_{c \in t c} \frac{\operatorname{numNegCont}(c, s g)}{\text { totalNumCont }} \times \operatorname{typeNegCont}(c)
\end{aligned}
$$

where numPosCont $(c, s g)$ represents the number of positive contributions of the type $c$ the configuration has to the softgoal $s g$, while typePosCont $(c)$ is the weight of the 
positive type of the contribution $c$. Analogously, numNegCont(c,sg) represents the number of negative contributions of the type $c$ the configuration has to the softgoal $\mathrm{sg}$, while typeNegCont(c) is the weight of the negative type of the contribution $c$. The variable totalNumCont represents the total number of contributions to the softgoal in the configuration.

The weight for each type of contribution is given in Table 2. In $i^{*}$-orthogonal, positive contributions are: Make, Help, Some+ and Unknown; the negative ones are: Break, Hurt and Some-.

Table 2. Weight of contribution link types

\begin{tabular}{|l|c|c|c|c|}
\hline Contribution & Make or Break & Help or Hurt & Some+ or Some- & Unknown \\
\hline Weight & 1,00 & 0,75 & 0,50 & 0,25 \\
\hline
\end{tabular}

After calculating the priority for each variant, the variant that should be selected is the one with the highest value for the priority function. If more than one variant has the highest value, our AE process implementation selects the first variant it used to calculate the priority function. This is done for each VP that needs to be prioritized. In the end of the prioritization, the new configuration of the DSPL is the one in which all VPs are bound to the variants that have the highest priority value.

Calculating the priority of Suggest Home Cooked Meal, find its value to be 6, while the priority value for Suggest Restaurant Meal is -6, since they have contributions only to the Save Money softgoal and these contributions are the exact opposite of each other (help and hurt). Therefore, the selected variant for current context is the Suggest Home Cooked Meal task.

Feature Configuration. This is an optional activity; it is executed if the DSPL's feature model was created in the Domain Engineering process. Mandatory features are included and the traceability table, created in the Create Feature Model activity, is used to select the optional features that are related to the $i^{*}$-orthogonal elements of the configuration. Since we did not generate a feature model for our running example, this activity is not executed.

The steps of the "Contextual Configuration" and "Variant Prioritization" activities are implemented and a rudimentary tool [Guedes 2020]. It does not have a graphic interface, nor is it integrated with GCL-Tool. This tool was created to select a configuration based on context variable values in order to perform the comparison published in Guedes, Silva and Soares (2017).

Guedes, Silva and Soares (2017) presented a comparison of ConG4DaS and other DSPL approaches regarding some modelling capabilities and a simulation-based assessment, comparing the configuration processes of ConG4DaS and REFAS (Requirements Engineering For self-Adaptive Software systems) [Muñoz-Fernández et al. 2015]. The results of the comparison show that ConG4DaS consistently selects configurations that maximize the satisfaction of softgoals considering the contributions of all variation points. On the other hand, REFAS generates configurations that do not maximize softgoals' satisfaction for all VPs and it is up to the domain analyst to choose the configuration that better satisfy the softgoals. 


\section{Survey Evaluation}

In this section, we evaluate ConG4DaS with respect to its perceived usefulness, using the Technology Acceptance Model (TAM) [Davis 1989]. According to Davis [Davis 1989], "people tend to use or not use an application to the extent they believe it will help them perform their job better". He refers to this as perceived usefulness. Then, Davis [Davis 1989] also defines the perceived ease of use as "the degree to which a person believes that using a particular system would enhance his or her job performance".

We used a survey to evaluate the perceived usefulness of ConG4DaS DE process for modelling DSPL variability and the perceived usefulness of ConG4DaS AE process for configuring DSPL variability. We asked the participants to read the description of ConG4DaS activities in its website [Guedes 2020], and then answer a questionnaire. Since we did not require the respondents to use ConG4DaS applying it to an example, they only had to read its full description, we could not evaluate its perceived ease of use. The only aspect of ease of use we evaluated was with respect to the necessity of training.

First, we performed a pilot study in order to get feedback about the length and readability of the questionnaire and ConG4DaS description. After making some modifications to the questionnaire and to the description of ConG4DaS activities [Guedes 2020], we sent it to the researchers of LER (in Portuguese, Laboratório de Engenharia de Requisitos - Requirements Engineering Lab) and the DSPL researchers, who were the authors of the 54 selected studies from our systematic mapping about variability management in DSPL [Guedes et al. 2015].

We used Google Forms, but we created two copies of the same questionnaire: one was sent to RE researchers and the other to DSPL researchers. Thus, the answers of both groups were separated and we could check if there were differences between the two groups. The questionnaire was available for the participants during 6 weeks, counting from the day the e-mails were sent. There were 8 respondents from around 40 researchers in LER mailing list (we call them the RE group). Only 7 DSPL researchers, from over one hundred authors to whom the e-mail was sent, answered the survey (we call them the DSPL group). The answers are anonymous so that the participants cannot be identified.

The questions of this survey and the detailed answers from all participants can be found in Guedes (2020). Note that some of the comments mention "guidelines" because the description we sent to the participants had not been updated with the new terminology, which uses the term "step".

\subsection{Participants' Experience}

The first set of questions, Phase 1, was about the participants' experience with Requirements Engineering, Dynamic Software Product Lines and Adaptive Systems.

1: What is your position in the Requirements Engineering area? Among the RE researchers, 3 are $\mathrm{PhD}$ students, 2 are professors, 2 are researchers and 1 is a former student of the RE area. From the DSPL researchers, 5 are professors and 2 are $\mathrm{PhD}$ students in the RE area. 
2: How many years of experience do you have with Requirements Engineering? Among the RE group, $50 \%$ have more than 5 years of experience in the RE area, $25 \%$ have 2 years of experience, $12.5 \%$ have 3 years and another $12.5 \%$ have 4 years of experience. Among the DSPL researchers, $71.4 \%$ have more than 5 years of experience with RE, $14.3 \%$ have 2 years and another $14.3 \%$ have 1 year.

3: Are you familiar with the $i^{*}$ framework? As it was expected, all LER respondents are familiar with $i^{*}$ models, half of them has used it outside a RE course, while the other half has not. Three of the DSPL researchers are not familiar with $i^{*}$, two of them can understand it, but have never used it, while the other two have used it outside a RE course.

4: What is your position in the Dynamic Software Product Lines area? In the RE group, there are 3 people that have not worked with DSPL, 2 are DSPL re-searchers, 2 are PhD students and 1 is an MSc student in the DSPL area. In the DSPL group, there are 4 professors, $2 \mathrm{PhD}$ students and 1 researcher.

5: How many years of experience do you have with Dynamic Software Product Lines? Most of the RE group has no experience with DSPL, one of them has 2 years of experience and another has less than 1 year of experience with DSPL. The experience of the DSPL group varies among 1 year $(14.3 \%), 2$ years $(14.3 \%), 3$ years $(14.3 \%), 4$ years $(14.3 \%), 5$ years $(14.3 \%)$ and more than 5 years $(28.6 \%)$.

6: What is your position in the Adaptive Systems area? In the RE group, 3 of them have no position in the Adaptive Systems area, 2 are researchers in the area, 2 are $\mathrm{PhD}$ students and 1 is an MSc student. In the DSPL group, there are 4 professors, $2 \mathrm{PhD}$ students and 1 researcher.

7: How many years of experience do you have with Adaptive Systems? Most participants from the RE group (62.5\%) have no experience with Adaptive Systems, 1 has more than 5 years of experience, 1 has 4 years and another one has 3 years of experience. In the DSPL group, most of them (71.4\%) have more than 5 years of experience, 1 has 4 years and the last has 1 year of experience with Adaptive Systems.

According to the answers of Phase 1, all participants have at least 1 year of experience with Requirements Engineering (see question 2). Everyone from the RE group has used i*, but only two of the DSPL have used it (see question 3). Most of the RE group does not have any experience with DSPL or Adaptive Systems, while all DSPL group have at least 1 year of experience in both areas (see questions 5 and 7).

\subsection{ConG4DaS DE Process Evaluation}

Phase 2 of the questionnaire was related to the perceived usefulness of the DE process of ConG4DaS. There were 6 affirmatives and the participants had to choose if they agreed with the affirmatives. The answer options were in Likert-scale. For some affirmatives, the participants could comment their answers, but this was optional. At the end of Phase 2, there was an optional question asking if the participant would like to make any comment on the DE process.

8: Goal Models, enriched with context, are useful for modelling variability of Dynamic Software Product Lines (DSPLs). Most of the RE group (62.5\%) agrees, 25\% of them strongly agree and $12.5 \%$ are undecided. $57.1 \%$ from the DSPL group agree, $14.3 \%$ strongly agree, $14.3 \%$ disagree and $14.3 \%$ are undecided. 
9: ConG4DAS' $i{ }^{*}$-orthogonal and context models are useful for modelling variability of DSPLS. Half of the RE group agrees and the other half strongly agrees. Most of the DSPL group (57.1\%) agrees, 28.6\% of them are undecided and $14.3 \%$ disagree.

10: ConG4DAS' $i *$-orthogonal and context models are useful for specifying the relationship between contexts and DSPL variability. Half of the RE group agrees, $37.5 \%$ strongly agree and $12.5 \%$ are undecided. Everybody in the DSPL agrees with the affirmative.

11: ConG4DAS' priority table is useful for specifying the relationship of contexts and softgoals' priority. Most of the RE group (62.5\%) strongly agrees with this affirmative, $25 \%$ are undecided and $12.5 \%$ agree. Most of the DSPL group $(71.4 \%)$ is undecided, while 2 of them agree.

12: The description of each mandatory activity is clear and easy to understand. The majority of the RE group (62.5\%) agrees with the affirmative and $37.5 \%$ of them strongly agree. $42.9 \%$ of the DSPL group are undecided, $28.6 \%$ of them agree, $14.3 \%$ disagree and $14.3 \%$ strongly disagree.

13: You would need training to execute ConG4DAS' mandatory activities. Half of the RE group disagrees, $37.5 \%$ of them strongly agree and $12.5 \%$ agree. In the DSPL group, $42.9 \%$ strongly agree, $28.6 \%$ are undecided, $14.3 \%$ agree and $14.3 \%$ disagrees.

14: Do you have any additional comments in regard to the DE process? Table 3 presents part of the comments from the RE group for this question, while Table 4 shows the answers from the DSPL group.

Table 3. Answers from RE group to question 14

\begin{tabular}{l} 
"Guidelines are very useful to product the models in each the \\
activities [...] However, it might require a lot of effort and more \\
amenable to induce errors without the aid of Case tool in these \\
activities" \\
"It is necessary guidelines to assign priorities to softgoals" \\
\hline
\end{tabular}

Table 4. Answers from DSPL group to question 14

"Experience with large and real RE issues in DSPL to demonstrate scalability"

"I would have liked to see more examples explaining the DE process in action"

\subsection{ConG4DaS AE Process Evaluation}

Phase 3 of the questionnaire was related to the perceived usefulness of the Ap-plication Engineering process of ConG4DaS. There were 4 affirmatives and one question for the participants to choose if they agreed with them, the choices were given in Likert-scale. 
Similar to Phase 2, there was also an optional space for the participants to comment their answers.

15: ConG4DAS' $i^{*}$-orthogonal and context models are useful for configuring Dynamic Software Product Lines. The majority of the RE group (62.5\%) agrees with this affirmative, $25 \%$ of them strongly agree and $12.5 \%$ are undecided. Most of the DSPL group (57.1\%) agrees and the other $42.9 \%$ are undecided.

16: ConG4DAS' AE activities are useful for configuring Dynamic Software Product Lines. Half of the RE group agrees with the affirmative, 25\% from this group strongly agree and $25 \%$ are undecided. Most of the DSPL group (57.1\%) agrees and the other $42.9 \%$ are undecided.

17: The description of each activity from the AE process is clear and easy to understand. Most of the RE group (62.5\%) agrees with the affirmative, 25\% of them strongly agree and $12.5 \%$ are undecided. In the DSPL group, $42.9 \%$ disagree with the affirmative, $28.6 \%$ agree and $28.6 \%$ are undecided.

18: The AE process occurs in execution time and it is, therefore, automatically executed. Given this fact, would you consider it is worth the effort of performing the mandatory activities of the DE process to obtain the configurations selected by the AE process? Half of the RE group agrees, $37.5 \%$ strongly agree and $12.5 \%$ are undecided. The majority of the DSPL group (57.1\%) agrees, 28.6\% are undecided and $14.3 \%$ strongly disagree.

19: Do you have any additional comments in regard to the AE process? Table 5 presents the comments from the RE group. Only one person from the DSPL group answered this question: "My answer is undecided to question 18 because the design time effort might not be worth it when requirements and user preferences change".

Table 5. Answers from RE group to question 19

"In addition to ranking the highest priority variant, maybe it is
interesting to consider a minimum threshold for variant
activation. Maybe a combination of contexts may lead to various
goals not been met, and in this situation some features should be
deactivated (instead of keep going with less harmful ones)"
"It's not so clear what are the cost-benefits of performing the DE
activities. Indeed, SPL domain engineering phase requires an up-
front investment. However, it would be interesting to measure this
effort in the context of this proposal in order to assess the benefits
of performing this phase when conducting the AE phase"

\subsection{Discussion}

Table 6 summarizes the answers of the RE group to the affirmatives in Phase 2 related to the perceived usefulness of ConG4DaS DE process. The abbreviations used on the tables of this section are: SA (strongly agree), A (agree), U (undecided), D (disagree) and SDA (strongly disagree). Note that $90 \%$ of the answers are positive - strongly agree $(42.5 \%)$ and agree $(47.5 \%)$ - and only $10 \%$ are neutral. It indicates that the participants of the RE group perceive ConG4DaS DE process as useful for modelling the variability of DSPLs. 
Table 7 summarizes the answers of the DSPL group regarding the affirmatives in Phase 2. As described in the table, most answers (57.14\%) are positive - strongly agree $(2.86 \%)$ and agree $(54.28 \%)-, 31.43 \%$ are neutral and $11.43 \%$ are negative disagree $(8.57 \%)$ and strongly disagree $(2.86 \%)$. It indicates that the participants of the DSPL group perceive ConG4DaS DE process as mostly useful, however, unlike the RE group, there are participants in this group that disagree.

Table 6. Answers of RE group for Phase 2

\begin{tabular}{|c|c|c|c|c|c|}
\cline { 2 - 6 } \multicolumn{1}{c|}{} & SA & A & U & D & SDA \\
\hline $\mathbf{8}$ & 2 & 5 & 1 & & \\
\hline $\mathbf{9}$ & 4 & 4 & & & \\
\hline $\mathbf{1 0}$ & 3 & 4 & 1 & & \\
\hline $\mathbf{1 1}$ & 5 & 1 & 2 & & \\
\hline $\mathbf{1 2}$ & 3 & 5 & & & \\
\hline Total & 17 & 19 & 4 & 0 & 0 \\
& $(42.5 \%)$ & $(47.5 \%)$ & $(10 \%)$ & $(0 \%)$ & $(0 \%)$ \\
\hline
\end{tabular}

Table 7. Answers of DSPL group for Phase 2

\begin{tabular}{|c|c|c|c|c|c|}
\cline { 2 - 6 } \multicolumn{1}{c|}{} & SA & $\mathbf{A}$ & $\mathbf{U}$ & $\mathbf{D}$ & SDA \\
\hline $\mathbf{8}$ & 1 & 4 & 1 & 1 & \\
\hline $\mathbf{9}$ & & 4 & 2 & 1 & \\
\hline $\mathbf{1 0}$ & & 7 & & & \\
\hline $\mathbf{1 1}$ & & 2 & 5 & & \\
\hline $\mathbf{1 2}$ & & 2 & 3 & 1 & 1 \\
\hline Total & 1 & 19 & 11 & 3 & 1 \\
& $(2.86 \%)$ & $(54.28 \%)$ & $(31.43 \%)$ & $(8.57 \%)$ & $(2.86 \%)$ \\
\hline
\end{tabular}

Regarding whether the description of the DE activities is clear and easy to understand (affirmative 12), everyone from the RE group agrees (3 strongly agree and 5 agree). But in the DSPL group, three of them are undecided, 2 agree, 1 disagrees and another strongly disagrees. We are not sure whether the differences in the answers from both groups are due to the fact that the participants from the RE group are familiar with $i^{*}$ models, while most of the DSPL is not. We need to investigate if the description of the steps is unclear or whether a more detailed ex-planation of the models is necessary.

Regarding the necessity of training to execute the DE process (affirmative 13), half of the RE group agrees that it is necessary, while the other half disagrees. In the DSPL group, most of them agree (3 strongly agree and 1 agrees), two are un-decided and one disagrees. But those who disagree in both groups are familiar with $i^{*}$ models, so we are not sure whether the required training is on the application of ConG4DaS or on the goal model used.

We did not include the results of affirmative 13 in Tables 6 and 7 because training is not related to the perceived usefulness, but to perceived ease of use. Moreover, the direction of the answers is reversed, i.e., when someone agrees that training is necessary, this answer is negative (it means the approach is difficult to use), while the disagreement is a positive answer (it means the approach is easy to use). We kept affirmative 13, though it is related to the perceived ease of use, because that 
particular aspect can be evaluated without using approach, solely based on the participants experience and by analysing the models and activities of ConG4DaS.

In regard to the perceived usefulness of ConG4DaS AE process, Table 8 summarizes the answers of the RE group. Most answers $(84.38 \%)$ are positive strongly agree $(28.12 \%)$ and agree $(56.25 \%)$ - and $15.62 \%$ are neutral. It indicates that the participants of the RE group perceive ConG4DaS AE process as useful for configuring DSPLs.

Table 8. Answers of RE group for Phase 3

\begin{tabular}{|c|c|c|c|c|c|}
\cline { 2 - 6 } \multicolumn{1}{c|}{} & SA & A & U & D & SDA \\
\hline $\mathbf{1 5}$ & 2 & 5 & 1 & & \\
\hline $\mathbf{1 6}$ & 2 & 4 & 2 & & \\
\hline $\mathbf{1 7}$ & 2 & 5 & 1 & & \\
\hline $\mathbf{1 8}$ & 3 & 4 & 1 & & \\
\hline Total & 9 & 18 & 5 & 0 & 0 \\
& $(28.13 \%)$ & $(56.25 \%)$ & $(15.62 \%)$ & $(0 \%)$ & $(0 \%)$ \\
\hline
\end{tabular}

Table 9 summarizes the answers of the DSPL group with respect to the affirmatives in Phase 3 . Half of the answers are positive - agree (50\%) - , 35.72\% are neutral and $14.28 \%$ are negative - disagree $(10,71 \%)$ and strongly disagree $(3,57 \%)$. It indicates that the participants of the DSPL group perceive ConG4DaS AE process as mostly useful, however, there is not such a convergence of opinions as in the RE group.

Table 9. Answers of DSPL group for Phase 3

\begin{tabular}{|c|c|c|c|c|c|}
\cline { 2 - 6 } \multicolumn{1}{c|}{} & SA & A & U & D & SDA \\
\hline $\mathbf{1 5}$ & & 4 & 3 & & \\
\hline $\mathbf{1 6}$ & & 4 & 3 & & \\
\hline $\mathbf{1 7}$ & & 2 & 2 & 3 & \\
\hline $\mathbf{1 8}$ & & 4 & 2 & & 1 \\
\hline Total & 0 & 14 & 10 & 3 & 1 \\
& $(0 \%)$ & $(50 \%)$ & $(35.72 \%)$ & $(10,71 \%)$ & $(3.57 \%)$ \\
\hline
\end{tabular}

The results of the survey were mostly positive. There were respondents with different background, one group with more experience in Requirements Engineering and another with more experience in DSPL and Adaptive Systems. How-ever, we are not able to generalize these results because the sample size is small, only 15 researchers answered the survey. Additionally, most participants may have only academic knowledge on RE and DSPL, i.e., they may not represent the opinion of specialists from industry.

Moreover, the results for affirmative 13, regarding the necessity of training, indicates that the adoption of ConG4DaS depends on training and may be also on experience with goal models. It is possible that companies that do not use goal models may resist the adoption of ConG4DaS. However, these adoption challenges might be overcome with training and tool support. 


\subsection{Threats of Validity}

In this section, we discuss some identified threats to the validity of this study. We believe the main threats are related to external, construct and internal validities. According to Wohlin et al. (2012), the external validity is concerned with to what extent it is possible to generalize the findings. Since only 15 researchers answered the survey, we are not able to generalize the findings. We sent the survey to all authors of the 54 studies of our systematic mapping on DSPL variability management, but only 7 responded. Additionally, the also sent it to a mailing list of RE researchers and obtained 8 answers.

For a future study, it should be considered to send it to authors who have published in venues related to both DSPL and RE. The focus should be in the latest 3 years, in order to get a more updated list of authors and their e-mails.

The construct validity concerns to what extent the operational measures that are studied really represent what the researcher has in mind and what is investigated according to the research questions [Wohlin et al. 2012]. One possible threat to this aspect of validity is whether the questions of the survey reflect the perceived usefulness, which is what we were trying to evaluate. We tried to mitigate such threat by using TAM [6], adapting the questions to our approach. We also performed a pilot study with former master and $\mathrm{PhD}$ students of our research group, to get their feedback regarding the questionnaire and ConG4DaS website.

According to Wohlin et al. (2012), threats to internal validity are influences that can affect the independent variable without the researcher's knowledge. One possible factor that could affect the respondents' perception of ConG4DaS was their experience with the models used by it. Therefore, we added a question related to the experience with iStar models, in order to take this aspect into account when discussing the results. Indeed, the respondents with some experience with iStar models (the RE group in particular), gave more positive answers.

We also added a page explaining $i^{*}$-orthogonal elements in ConG4DaS website so that the respondents could read it if they thought necessary. However, reading the material about $i^{*}$-orthogonal was not a mandatory for taking the survey. In a future study, a basic training on the models used by ConG4DaS should be considered.

\section{Conclusion}

In this paper, we presented ConG4DaS, a RE approach for DSPL that models variability using contextual goal models that includes goals, contexts and NFRs (as softgoals). The configuration process uses this information for selecting a product configuration that satisfies current context and maximizes NFRs satisfaction according to their priority.

We evaluated ConG4DaS with respect to its perceived usefulness through a survey with RE and DSPL researchers. A total of 15 researchers answered the survey and most of them perceived ConG4DaS DE process as useful for modelling DSPL's variability $-90 \%$ of positive answers from the RE group and $57.14 \%$ from the DSPL group. Regarding the AE process, most respondents also found it useful $-84.38 \%$ of positive answers from the RE group and 50\% from the DSPL group.

As future work, we intend to provide integrated tool support for all activities of ConG4DaS, since the $\mathrm{DE}$ and $\mathrm{AE}$ processes are supported by different tools. 
Additionally, we intend to evaluate the perceived ease of use of ConG4DaS, by asking a group of DSPL specialists to apply the approach to an example and, then, ask them to answer a survey.

\section{References}

Ali, R., Dalpiaz, F. and Giorgini, P. (2013). "Reasoning with contextual requirements: Detecting in-consistency and conflicts", In: Information and Software Technology. $55,35-57$.

Bencomo, N., Hallsteinsen, S. and Almeida, E. (2012). "A View of the Dynamic Software Product Line Landscape", In: Computer. 45, 36-41.

Bencomo, N., Sawyer, P., Blair, G. S. and Grace, P. (2008). "Dynamically Adaptive Systems are Product Lines too: Using Model-Driven Techniques to Capture Dynamic Variability of Adaptive Systems", Paper presented at The 2nd International Workshop on Dynamic Software Product Lines (DSPL 2008), with SPLC 2008, Limerick, Ireland,.

Capilla, R., Bosch, J., Trinidad, P., Ruiz-Cortés, A. and Hinchey, M. (2014). "An overview of Dynamic Software Product Line architectures and techniques: Observations from research and industry", In: Journal of Systems and Software. 91, 3-23.

Clements, P. and Northrop, L. (2001). "Software Product Lines: Practices and Patterns", 1st ed. Addison-Wesley Longman Publishing Co., Boston, MA, USA.

Davis, F. (1989). "Perceived Usefulness, Perceived Ease of Use, and User Acceptance of In-formation Technology", In: MIS Quarterly. 13, 319.

Grau, G., Franch, X. and Maiden, N. (2008). "PRiM: An i*-based process reengineering method for information systems specification", In: Information and Software Technology. 50, 76-100.

Guedes, G. (2020). ConG4DaS website, https://sites.google.com/site/cong4das/, last accessed 2020/12/15.

Guedes, G., Silva, C., Soares, M., and Castro, J. (2015). "Variability Management in Dynamic Software Product Lines: A Systematic Mapping”, In: 2015 IX Brazilian Symposium on Components, Architectures and Reuse Software. Belo Horizonte, Brazil.

Guedes, G., Silva, C. and Soares, M. (2017). "Comparing Configuration Approaches for Dynamic Software Product Lines", In: Proceedings of the 31st Brazilian Symposium on Software Engineering - SBES'17. Fortaleza, Brazil.

Hallsteinsen, S., Hinchey, M., Park, S. and Schmid, K. (2008). "Dynamic Software Product Lines", In: Computer. 41, 93-95.

Hinchey, M., Park, S. and Schmid, K. (2012). "Building Dynamic Software Product Lines", In: Computer. 45, 22-26.

Kotonya, G. and Sommerville, I. (1998). "Requirements Engineering: Processes and Techniques", 1st ed. Wiley Publishing, England. 
Lamsweerde, A. (2001). "Goal-oriented requirements engineering: a guided tour", In: Proceedings Fifth IEEE International Symposium on Requirements Engineering. Toronto, Canada.

Liaskos, S., Lapouchnian, A., Yu, Y., Yu, E. and Mylopoulos, J. (2006) "On Goalbased Variability Acquisition and Analysis", In: 14th IEEE International Requirements Engineering Conference (RE'06). Minneapolis/St. Paul, USA.

Lima, C. (2011). "E-SPL - a approach for requirements phase in domain engineering and application engineering with goal models" (in Portuguese: "E-SPL - uma abordagem para a fase de requisitos na engenharia de domínio e na engenharia de aplicação com modelos de objetivos"). Master's thesis. Informatics Center (CIn), UFPE, Brazil.

Muñoz-Fernández, J. C., Tamura, G., Mazo, R. and Salinesi, C. (2015) "Towards a Requirements Specification Multi-View Frame-work for Self-Adaptive Systems", In: CLEI Electronic Journal. 18, 2.

Pimentel, J., Lucena, M., Castro, J., Silva, C., Santos, E. and Alencar, F. (2012). "Deriving software architectural models from requirements models for adaptive systems : the STREAM-A approach", In: Requirements Engineering, v. 17, p. 259281. DOI: 10.1007/s00766-011-0126-z.

Pohl, K., Böckle, G. and van der Linden, F. J. (2005). "Software Product Line Engineering: Foundations, Principles and Techniques", Springer-Verlag New York, Inc., Secaucus, NJ, USA.

Sommerville, I. (2010). "Software Engineering", 9th ed. Addison-Wesley Publishing Company, USA.

Varela, J. (2015). "Using Contexts and Non-functional Requirements for Configuring Goal Models, Feature Models and Scenarios of Software Product Lines" (in Portuguese: "Usando contextos e Requisitos Não-Funcionais para Configurar Modelos de Objetivos, Modelos de Features e Cenários para Linhas de Produto de Software"). Master's thesis. Informatics Center (CIn), UFPE, Brazil.

Wohlin, C., Runeson, P., Höst, M., Ohlsson, M. C., Regnell, B. and Wesslén, A. (2012). "Experimentation in software engineering", Springer-Verlag New York, New York.

Yu, E., Giorgini, P., Maiden, N., Mylopoulos, J. and Fickas, S. (2011). "Modeling Strategic Relationships for Process Reengineering", In: Social Modeling for Requirements Engineering. 1st ed., MIT Press, pp.11-152. 